\title{
EVALUACIÓN DE VARIABLES DASOMÉTRICAS DE REBROTES EN Eucalyptus camaldulensis DEHNH, EN LA FINCA EL PLANTEL, NINDIRI, MASAYA
}

\section{EVALUATION OF DASOMETRIC VARIABLES OF SPROUTS OF Eucalyptus camaldulensis DEHNH, AT THE EXPERIMENTAL FARMS EL PLANTEL, NINDIRI, MASAYA}

\author{
Reyes Flores Francisco Giovanni', Membreño Morales Juan José1, Yelsy Adlleris Chávez Lovo² \\ ${ }^{1}$ Ing. MSc. Docentes de la Facultad de Recursos Naturales y del Ambiente (FARENA) \\ ${ }^{2}$ Ing. Graduada de la Carrera de Ingeniería Forestal
}

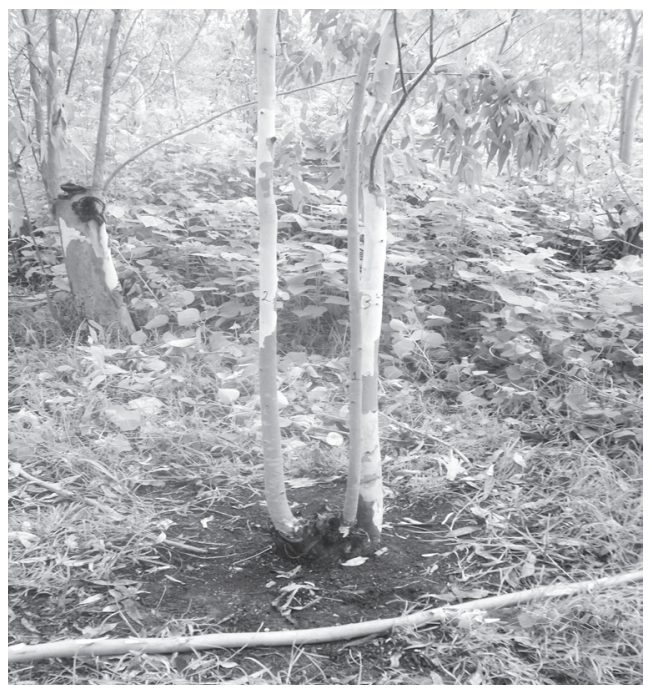

\section{RESUMEN}

El estudio de evaluación de la capacidad de rebrote de Eucaliptus camaldulensis Dehnh, tuvo como objetivo evaluar el comportamiento de los rebrotes en plantaciones a través de la aplicación de tratamiento silviculturales de poda en áreas que han sido aprovechadas. La metodología consistió en establecer un recorrido de campo en la plantación de eucalipto en la unidad experimental finca El Plantel, con el propósito de seleccionar áreas para el establecimiento de parcelas en áreas que habían sido aprovechadas anteriormente. Una vez seleccionadas las parcelas se procedió a la selección de los rebrotes, de los cuales se dejaron los más vigorosos y que tuviesen un fuste recto, se seleccionaron los tres mejores ejes de los tocones. La toma de datos en el campo se realizó evaluando las siguientes variables: altura del tocón mayor de $20 \mathrm{~cm}$, altura del tocón menor de $20 \mathrm{~cm}$, diámetro del tocón de $15 \mathrm{~cm}$, y diámetro del tocón entre 15 a $20 \mathrm{~cm}$. Los tratamientos silviculturales tuvieron influencia en el incremento del crecimiento en diámetro y altura en los rebrotes, la limpieza de la maleza, eliminación de lianas y raleo de rebrotes, favoreciéndolos al no existir competencia para los rebrotes seleccionados. De acuerdo al análisis estadístico t-Studen la variable tocón menor de $20 \mathrm{~cm}$ de altura, es la mejor variable para la realización de futuro manejo de rebrotes. La sobrevivencia en las parcelas establecidas independientemente de las cuatro variables que se aplicaron, es buena según la clasificación utilizada en el experimento. Se recomienda seguir aplicando tratamientos silviculturales para un mejor rendimiento en el crecimiento tanto en diámetro y altura de los rebrotes.

\section{SUMMARY}

The assessment study of the capacity of regrowth of Eucalyptus camaldulensis Dehnh, is aimed to evaluate the behavior of sprouts into plantations by implementing pruning silvicultural treatment in areas that have been exploited. The methodology used consisted of establishing a field trip in the plantation of eucalyptus in the experimental farm el Plantel, for the purpose of selecting areas for establishing plots in areas that had been previously exploited. After selected the plots, we proceeded to the selection of the sprouts, from which, the most vigorous with straight trunk were selected, as well as, the best three axes of the stumps. Data collection in the field was performed by evaluating the following variables: stump height greater than $20 \mathrm{~cm}$, stump height less than $20 \mathrm{~cm}$, stump diameter of $5 \mathrm{~cm}$ and stump diameter between $15-20 \mathrm{~cm}$. Silvicultural treatments had an influence on the increase of height and diameter growth in the shoots, weed control, the remove of vines and thinning of sprouts, favoring them in the absence of competition for the selected sprouts. According to statistical analysis t Studen, the variable stump less than $20 \mathrm{~cm}$ in height, is the best performing variable for the future management of the shoots. The plants survival in the plots established independently of the four variables that were applied, is good according to the classification used in the experiment. It is recommended to continue applying silvicultural treatments for better performance in both diameter growth and height of the sprouts. 
$\mathrm{U}$ na importante característica del eucalipto es su facultad para brotar vigorosamente de la cepa o el tocón. En el caso de Eucalyptus camaldulensis pueden obtenerse sucesivos aprovechamientos de un mismo tocón o cepa. Un adecuado tratamiento de los brotes contribuye a la obtención de las siguientes cosechas.

El E. camaldulensis, es una especie identificada como prioritaria para la reforestación en el país, ya que tiene características que justifican su importancia como: rápido crecimiento, uso múltiples, lo que hace altamente deseable para su establecimiento. La capacidad de rebrotes tiene mucha importancia para el agricultor porque después de plantar un árbol puede manejarlo para obtener varios cortes si se implementan técnicas adecuadas de manejo (Valdivia, 2006)

El manejo de rebrotes es un sistema rápido, simple, aplicable sin grandes dificultades, tomando en cuentas que son empleadas a muchas especies que rebrotan y crean un recurso renovable donde antes no existía, obteniendo una ganancia que puede permitir un valor económico de vegetaciones consideradas sin valor (Valdivia, 2006)

En el presente estudio se evaluó el comportamiento de los rebrotes de eucalipto ante la aplicación del tratamiento silvicultural raleo de rebrotes, también se utilizará la eliminación de lianas, y cáseo del tocón, para así obtener información en un futuro que podrá ser utilizada para las personas interesadas en manejos más adecuados de las plantaciones (MARENA/INAFOR, 2002)

La importancia en los rebrotes es que se pueden utilizar en construcciones rústicas como madera en rollo, postes, estacas, construcciones, carpintería en general y artículos torneados. La madera de eucalipto cuando está completamente seca constituye un combustible excelente, produciendo leña y carbón de óptima calidad. Su poder calorífico es de $4800 \mathrm{Kcal} / \mathrm{kg}$. E. Camaldulensis es una especie apropiada para utilizarse en el establecimiento de cortinas rompe vientos, debido a su altura, permeabilidad de la copa, resistencia a los vientos, alta capacidad (MARENA/ INAFOR, 2002)

\section{MATERIALES Y MÉTODOS}

Origen de la especie e introducción en Nicaragua. La especie Eucalyptus camaldulensis Dennh es originaria de Australia, llego a Nicaragua hace unos 50 años, pero no tuvo mucha relevancia, tomando auge durante la década de los 80 , en la cual alcanzó popularidad, al emplearlo masivamente para formar cortinas rompe viento que controlan la erosión eólica en las peladas llenuras algodoneras del occidente de León y Chinandega (Fernández, s.f.).

Descripción del sitio. La finca El Plantel es una unidad de experimentación y evaluación, propiedad de la Universidad Nacional Agraria, situada en el kilómetro 31 carretera Tipi-
tapa-Masaya, en el municipio de Nindiri, departamento de Masaya, contiguo a la empresa Avícola Estrella (figura 1). Cuenta con un área de 270 manzanas, entre las coordenadas: $12^{\circ}-06^{\prime}-24^{\prime \prime}$ y $12^{\circ}-07^{\prime}-30^{\prime \prime}$ latitud norte y los $86^{\circ} 04^{\prime} 46^{\prime \prime}$ y $86^{\circ} 05^{\prime}$ 87" longitud oeste (Somarriba, 1989).

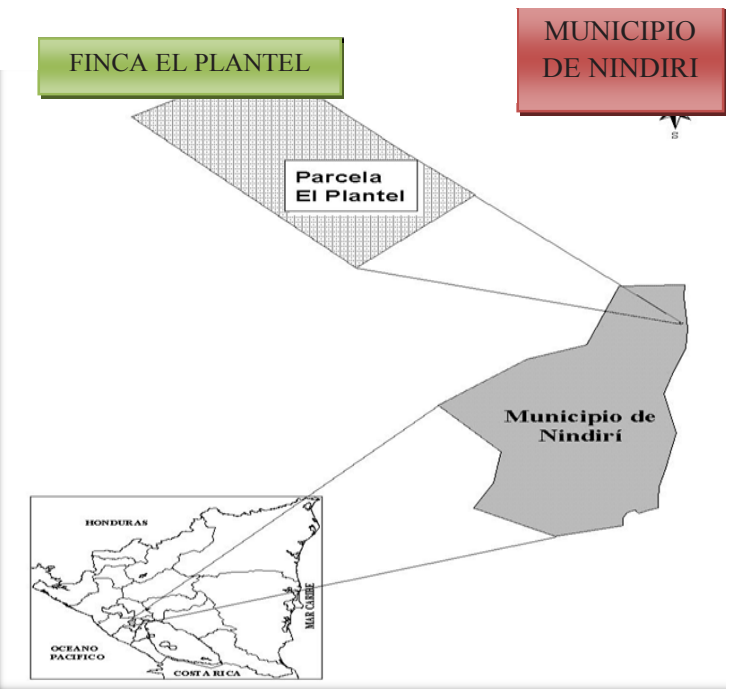

Figura 1. Ubicación de la unidad de experimentación de la Universidad Nacional Agraria, Nindiri, de Masaya.

Suelo. El área donde está localizada la finca El Plantel corresponde a una transición entre el bosque tropical, moderadamente denso y seco, y bosque tropical sub húmedo. Los factores formadores de los suelos son vulcanismo y tectonismo, los procesos formadores que han modelado son la erosión y la sedimentación. La textura varía desde arenosa a franco en términos generales se considera que los suelos son bien drenados con fertilidad aceptable (Somarriba, 1989).

Clima. De acuerdo con la clasificación de koppen, el clima es tropical de sabana con transición a sub -tropical, semi húmedo. La precipitación varía de $600 \mathrm{~mm}$ a $1800 \mathrm{~mm} / \mathrm{año}$. Existe una marcada época seca durante seis meses (noviembre-abril), durante la época seca, el promedio de precipitaciones es de 0-3 mm en los meses más secos. Los de mayor precipitación son los de septiembre y octubre en que ocurre un promedio de 200 a $250 \mathrm{~mm}$ de lluvia (Somarriba, 1989).

La temperatura mínima media corresponde al mes de diciembre con valores que varían entre $24.7^{\circ} \mathrm{C}$ y $25.2^{\circ} \mathrm{C}$. La evaporación medio anual es de $2044 \mathrm{~mm}$, el mes con mayores evaporaciones es abril. La variación mensual de la evaporación es opuesto a la marcha de precipitaciones y de los valores medios de la humedad relativa predominantes, independientes de su velocidad tiene velocidad promedio de $3.4 \mathrm{~m} / \mathrm{seg}$ (12 km hora) (Somarriba, 1989).

Vegetación. La formación de vegetación se caracteriza por desarrollarse bajo un régimen entre los 750-1 $250 \mathrm{~mm}$ a una 
temperatura entre 26 y $29^{\circ} \mathrm{C}$ ubicada en un rango de elevación que va de los 0 a $500 \mathrm{msnm}$, donde llueve de mayo a octubre. Esta vegetación fue eliminada con la introducción del monocultivo del algodón durante los años 50 y 60 . Actualmente la tierra se usan con cultivos de granos básicos como maíz, (Zea mays.) sorgo (Sorghum vulgare Pers) y frijol (Phaseolus vulgaris) además de frutales como cítricos, (Citrus sp) mangos (Mangifera indica L), aguacate (Persea americana mill.), plátanos (Musa sp), y yuca (Manihot esculenta) cultivo común en esta área (Somarriba, 1989).

\section{Metodología del trabajo}

Reconocimiento del área de estudio. Se realizó el recorrido de campo en la plantación de eucalipto en la unidad experimental finca El Plantel, con el propósito de seleccionar áreas para el establecimiento de parcelas en áreas que habían sido aprovechadas anteriormente (figura 2).

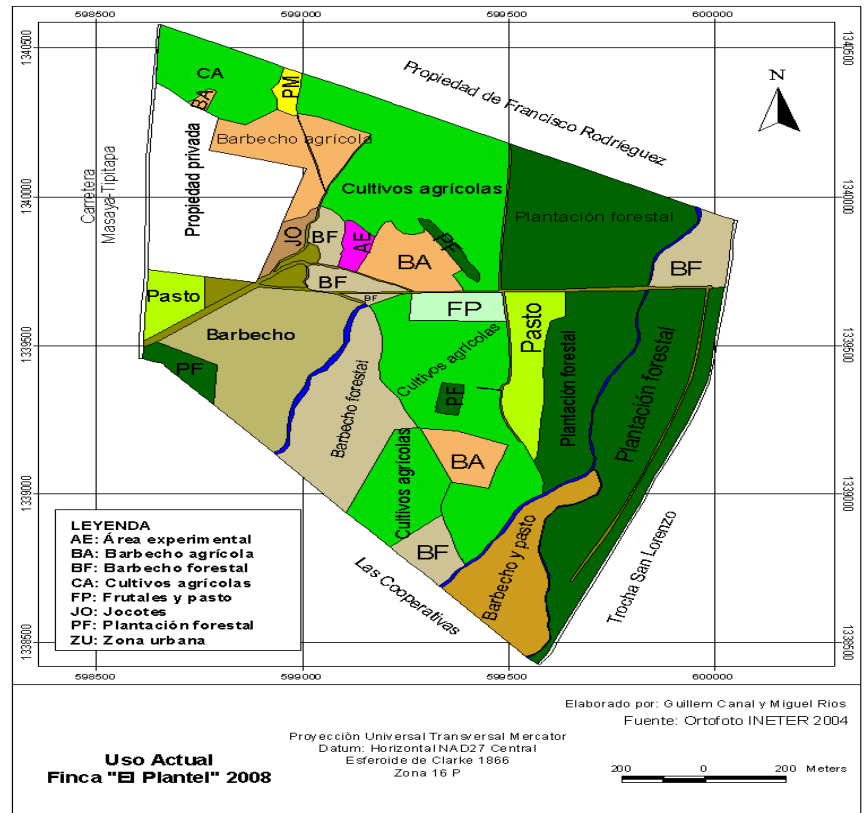

Figura 2. Mapa de ubicación de parcelas de medición en la finca El Plantel de la Universidad Nacional Agraria, Nindiri, Masaya 2011.

Selección de tocones muestreados. Se seleccionó tocones que cumplieran con los siguientes criterios de medición: altura del tocón mayor de $20 \mathrm{~cm}$, altura del tocón menor de 20 $\mathrm{cm}$, diámetro del tocón de $15 \mathrm{~cm}$ y diámetro del tocón entre 15 a $20 \mathrm{~cm}$ en cada una de las cuatro parcelas. Se eligieron 50 tocones, en total se seleccionó 200 tocones que cumplieran con los criterios establecidos para cada ensayo, buenas características fenotípicas y tener más de cuatro rebrotes.

Tratamientos silviculturales aplicados a los tocones seleccionado en cada parcela establecida. Una vez elegida el área, se establecieron las parcelas. A cada parcela se le aplicaron los siguientes tratamientos silviculturales.
Cáseo del tocón. Se realizó la limpieza de maleza al ras del suelo, con un distanciamiento de un metro de radio alrededor del tocón, considerando que el cáseo le dará mejor libertad de crecimiento, ya que la maleza compite con los tocones y rebrotes por nutriente, luz y espacio, lo que una vez eliminada le dará mejor ventaja de crecimiento al rebrote.

Eliminación de lianas. Esta actividad consistió en dejar limpio los rebrotes, ya que las lianas se enredan en los rebrotes, interfiriendo en su desarrollo.

Raleo de rebrotes. Se seleccionó los tres mejores rebrotes con altas posibilidades de sobrevivencia, observando el grosor del diámetro y la altura del rebrote. Se dejaron los rebrotes que estaban a favor de la dirección del viento, para evitar el desprendimiento del tocón.

\section{Diseño de parcelas}

Tamaño de la parcela y distanciamiento. El área total de la plantación de eucalipto es de 10.42 hectáreas, se seleccionaron cuatro parcelas. El área neta de las cuatro parcelas fue 1.608 ha, lo que representa el $15.43 \%$ del área total de la plantación.

El distanciamiento de los árboles es de $2 \mathrm{~m}$ x $2.80 \mathrm{~m}$ las dimensiones de cada parcela fueron de $200 \mathrm{~m}$ de largo por $8.40 \mathrm{~m}$ de ancho. El tamaño de cada parcela es de 0.168 ha con un callejón sin árboles de 14.90 metros de ancho entre cada una de las cuatro parcelas (figura 3 ).

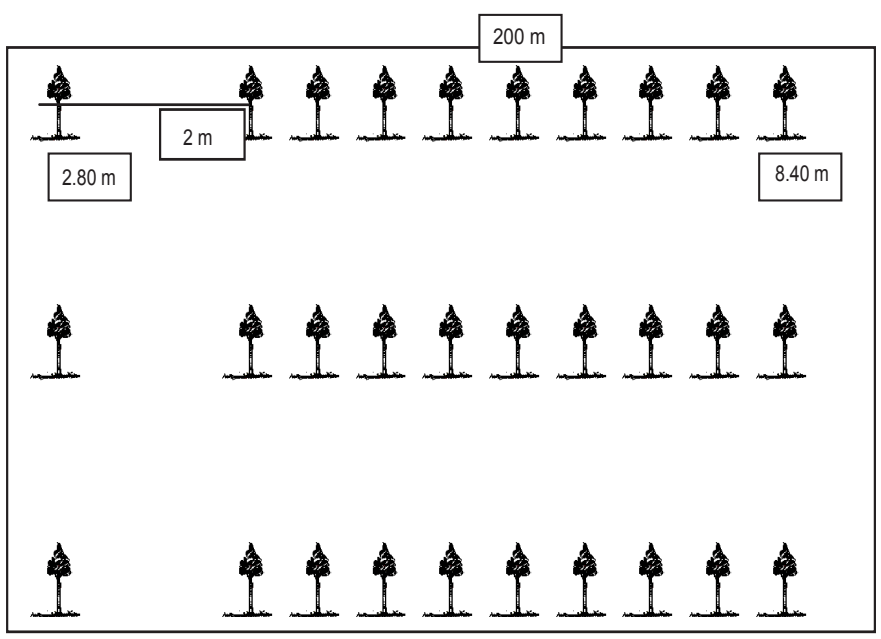

Figura 3. Diseño de la parcela de muestreo, finca El Plantel, Nindiri, Masaya 2011.

Selección de los rebrotes. En el área aprovechada se establecieron parcelas con tratamiento silvicultural, siendo este el raleo de rebrote. Las características principales de los rebrotes seleccionados fueron los dominantes, basándose en características morfológicas como fuste recto, vigoroso, sin daños mecánicos y sin incidencias de plagas. El número de rebrotes que se dejaron en el tocón fueron tres rebrotes, tomando en cuenta el diámetro y distribución en el tocón. 
Variables dasométricas a estudiar en los incrementos de rebrotes en la plantación de eucalipto. En cada uno de los tres rebrotes seleccionados por cada tocón, se tomaron los siguientes datos dasométricos (figura 4)
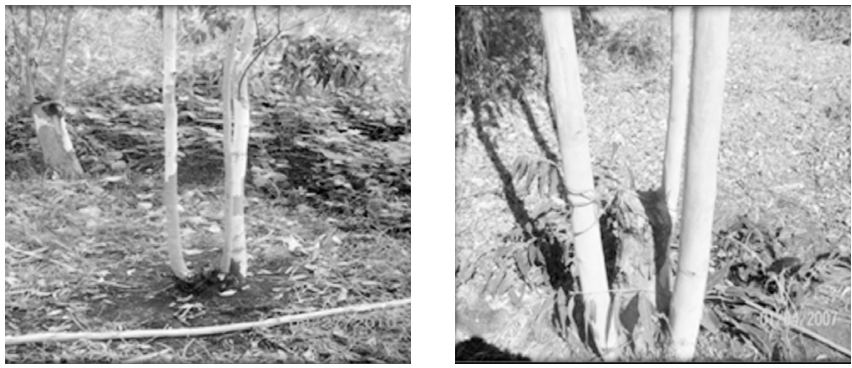

Figura 4 Selecccion de rebrotes en la finca El Plantel Universidad Nacional Agraria, Nindiri, Masaya, 2011.

Altura del rebrote. Para la medición de la altura de un rebrote se mide desde donde sale el rebrote hasta la punta del ápice a lo largo del fuste (ADEFOR, 1995). En el presente estudio la medición de altura se realizó en cada rebrote, recto y bien definido, desde la base donde sale el rebrote hasta el ápice del mismo. Dicha medición se realizó con una vara telescópica ayudado con una cinta métrica. (figura 5). La medición de altura y diámetro se realizaron en dos épocas: agosto 2010 (época de lluvia) y marzo 2011 (época seca) con una duración de siete meses.

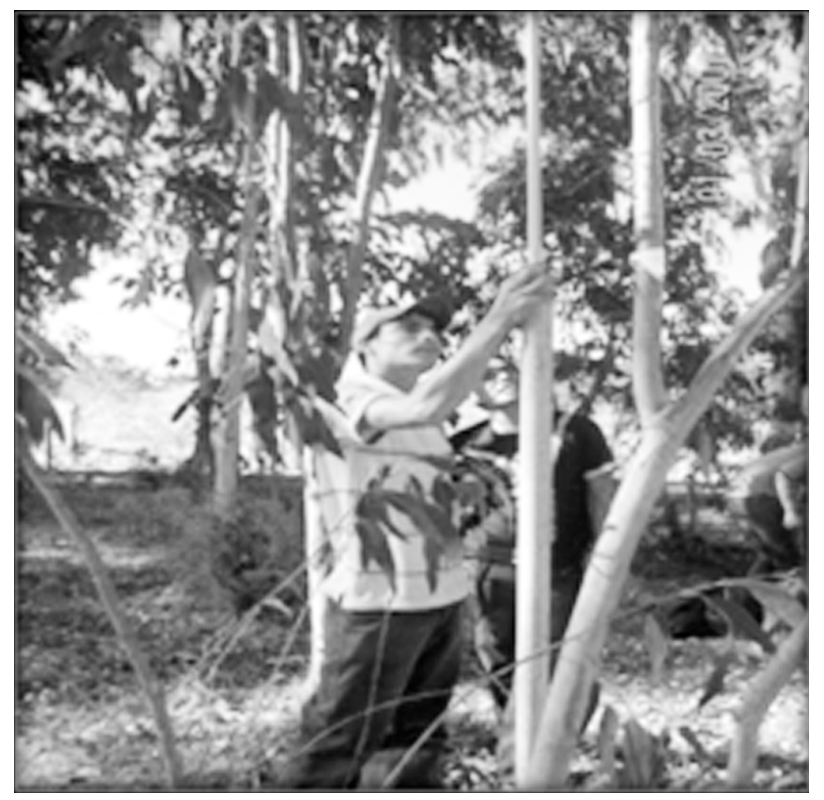

Figura 5. Medicion de altura de rebrotes en la finca El Plantel, Universidad Nacional Agraria, Nindiri, Masaya, 2011.
Diámetro del rebrote. Se procedió a realizar la medición y recolección de datos del diámetro del rebrote, estableciendo como medida $5 \mathrm{~cm}$ de altura después de la base del rebrote con una cinta diamétrica, en cada uno de los tres rebrotes que se dejaron en el tocón (Figura 6).

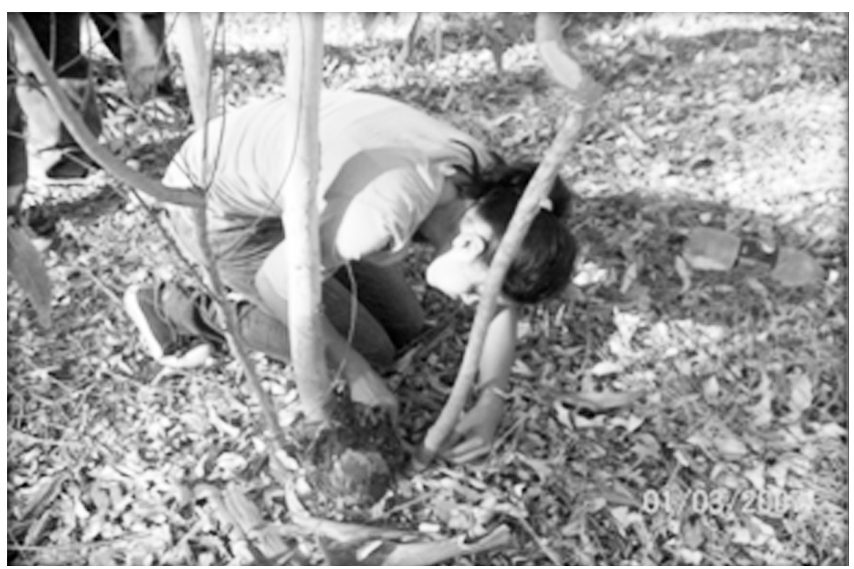

Figura 6. Medicion de diametro de rebrotes en la finca El Plantel Universidad Nacional Agraria, Nindiri, Masaya, 2011.

Descripción de los criterios empleados en la plantación de Eucalipto

Altura del tocón mayor de $20 \mathrm{~cm}$. En la selección de este tocón se midió con una cinta mitrica desde la superficie del suelo hasta sobrepasar los $20 \mathrm{~cm}$, con lo cual pasa a ser elegible dentro de la variable de la muestra.

Altura del tocón menor de $20 \mathrm{~cm}$. Se seleccionaron tocones que tuvieran una altura menor a $20 \mathrm{~cm}$.

Diámetro del tocón de $\mathbf{1 5} \mathrm{cm}$. Se realizó la medición del tocón que registrara un diámetro de $15 \mathrm{~cm}$, usando una cinta diamétrica con lo cual pasa a ser elegible.

Diámetro del tocón entre 15 a $20 \mathrm{~cm}$. Se tomaron los tocones que tuvieran un diámetro entre 15 y $20 \mathrm{~cm}$. utilizando cinta diamétrica, con lo cual pasa a ser elegible dentro de la variable de la muestra.

Sobrevivencia de rebrotes. La sobrevivencia se evaluó en base al número de rebrotes vivos que se observaron al final de la segunda medición realizada, utilizando la categorización de Centeno (1993) citado por González et al., (2008), quien indica que un porcentaje de sobrevivencia superior al $80 \%$ corresponde a categoría buena, entre 40 y $80 \%$ de sobrevivencia corresponde a categoría regular, y porcentaje de sobrevivencia menos de 40 por ciento corresponde a categoría mala.

Procesamiento de datos. Se realizó un levantamiento de datos o información cuantitativa en las parcelas en formato de registro. Recopilada la información se introdujo al programa estadístico de Excel, este programa facilitó las conversiones 
de la información. El análisis estadístico descriptivo determinó el incremento tanto en altura, como en diámetro de los rebrotes, obtenida esta información se realizó el análisis de t-Studen para determinar si hubo nivel de significancia y determinar cuál de las variables influyó en el crecimiento del diámetro y altura del rebrote.

Calculo del incremento promedio mensual (IPM) de rebrotes en altura y diámetro del Eucalyptus camaldulensis. En los rebrotes de eucalipto se determinó el incremento promedio mensual (IPM) para un periodo de siete meses, se consideró el total del incremento y se dividió por el periodo evaluado.

\section{RESULTADOS Y DISCUSIÓN}

Los datos obtenidos, tanto en la primera como en la segunda medición se introdujeron en un programa estadístico descriptivo, dando los siguientes resultados:

Altura del rebrote con altura del tocón mayor y menor de $20 \mathrm{~cm}$. En los rebrotes con tocones mayor de $20 \mathrm{~cm}$, se obtuvo un incremento promedio de 1.06 metros de altura en los tres rebrotes que se dejaron en cada uno de los tocones evaluados (tabla 1). La última medición se realizó a los sietes meses, obteniendo un incremento promedio mensual de $0.15 \mathrm{~m}$

En los rebrotes con tocones menores de $20 \mathrm{~cm}$, el incremento promedio de altura de los ejes fue de 2.41 metro, lo que representa un incremento promedio mensual de $0.34 \mathrm{~m}$.

Al realizarse comparaciones en estudio de incremento de altura en rebrotes de eucalipto, en diferentes comunidades del municipio de Telica, se encontró que en las Marías, se obtuvo un valor promedio de 1.21 metros de altura, en Los Mangles de 1.05 metros y La Carpa de un metro en un lapso de seis meses de evaluación (Pérez, 2004). Resulta que la finca El Plantel se registran resultados mayores en comparación con la comunidad Las Marías.

Observándose una diferencia mayor en incremento en la finca El Plantel, dicha diferencia está influenciada por la temperatura y el tipo de suelo de la micro cuenca Las Marías, ya que son suelos clasificados por su profundidad, textura superficial y del subsuelo así como por el grado de pedregosidad superficial. Mientras que en la finca El Plantel, el suelo es franco arenoso bien drenado y fertilidad aceptable. También es posible que haya influido el cáseo que se dio a cada tocón, el raleo de rebrotes nuevo y la limpieza de lianas.

Diámetro del rebrote con altura del tocón mayor de $20 \mathrm{~cm}$ y menor de $20 \mathrm{~cm}$. En el incremento del diámetro promedio de los rebrotes se observó un incremento de $0.79 \mathrm{~cm}$ con el tratamiento altura del tocón mayor de $20 \mathrm{~cm}$ (tabla 1). Al igual que la altura del rebrote, la última medición se realizó a los siete meses, obteniendo un incremento promedio mensual del diámetro de $0.11 \mathrm{~cm}$.
En el incremento promedio de diámetro en los rebrotes medidos se obtuvo aumento de $2.57 \mathrm{~cm}$ en el tratamiento altura del tocón menor de $20 \mathrm{~cm}$ observándose un incremento promedio mensual de $0.36 \mathrm{~cm}$, al cabo de 7 meses de diferencia entre cada medición.

Los incremento en diámetros de los rebrotes que se dio en el municipio de Telica, en las comunidades de Las Marías es de $1.4 \mathrm{~cm}$, en los Los Mangles de $1 \mathrm{~cm}$ y en La Carpa con $1.5 \mathrm{~cm}$ a los seis meses (Pérez, 2004). Nuevamente en la finca El Plantel, se obtuvieron mejores resultado al comprar la variable diámetro del tocón. Este resultado en la unidad experimental de la finca El Plantel se deba a los mismos criterios comentados para la altura del tocón.

Altura del rebrote con diámetro del tocón de $15-20 \mathrm{~cm}$ y de $15 \mathrm{~cm}$. Se alcanzó un incremento promedio de altura en los rebrotes de 2.23 metros en el tratamiento diámetro del tocón de 15 a $20 \mathrm{~cm}$ (tabla 1). El Incremento promedio mensual de la altura en este tratamiento en siete meses es de $0.31 \mathrm{~m}$. En el tratamiento diámetro del tocón menores de 15 $\mathrm{cm}$. el incremento promedio de altura de los rebrotes es de 2.65 metros, observándose un incremento promedio mensual de $0.37 \mathrm{~m}$.

Diámetro del rebrote con diámetro del tocón de $15 \mathrm{~cm}$ y de 15-20 cm. En el incremento del diámetro promedio se obtuvo un aumento de $2.80 \mathrm{~cm}$ en los rebrotes con el tratamiento diámetro de tocón $15 \mathrm{~cm}$ a los siete meses de evaluación. El incremento promedio mensual es de $0.40 \mathrm{~cm}$ (tabla 1).

El incremento promedio de diámetro de los rebrotes obtuvo un valor de $2.46 \mathrm{~cm}$ en el tratamiento diámetro tocón menor de 15 a $20 \mathrm{~cm}$. El incremento promedio mensual es de $0.35 \mathrm{~cm}$. Comparando los incrementos en los dos tipos de tratamientos implementado en la plantación de eucalipto, con respecto al diámetro del tocón es mínima. 
Tabla 1. Incremento en altura de los rebrotes por tipo de altura de tocón, diámetro de los rebrotes por tipo de altura del tocón, altura de los rebrotes por tipo de diámetro del tocón y diámetro de los rebrotes por tipo de diámetro del tocón, finca el Plantel, 2011

\begin{tabular}{|c|c|c|c|c|}
\hline & $\begin{array}{l}\text { Incremento } \\
\text { en altura }(\mathrm{m})\end{array}$ & $\begin{array}{l}\text { Incremento en } \\
\text { diámetro }(\mathrm{cm})\end{array}$ & $\begin{array}{l}\text { Incremento } \\
\text { en altura }(\mathrm{m})\end{array}$ & $\begin{array}{l}\text { Incremento en } \\
\text { diámetro }(\mathrm{cm})\end{array}$ \\
\hline$>20 \mathrm{~cm}$ & 1.06 & 0.79 & & \\
\hline Incremento promedio mensual (m) & 0.15 & 0.11 & & \\
\hline$<\operatorname{de~} 20 \mathrm{~cm}$ & 2.41 & 2.57 & & \\
\hline Incremento promedio Mensual $(\mathrm{cm})$ & 0.34 & 0.36 & & \\
\hline \multirow{2}{*}{\multicolumn{3}{|c|}{$\begin{array}{l}15-20 \mathrm{~cm} \\
\text { Incremento promedio mensual (m) }\end{array}$}} & 2.23 & 2.46 \\
\hline & & & 0.31 & 0.11 \\
\hline \multicolumn{3}{|l|}{$15 \mathrm{~cm}$} & 2.65 & 2.80 \\
\hline \multicolumn{3}{|l|}{ Incremento promedio mensual (m) } & 0.37 & 0.36 \\
\hline
\end{tabular}

Diámetro del rebrote. La prueba de tStudent se realizó una comparación de mediana del incremento del diámetro de los rebrotes, dando un resultado de 1.80 metros en tocones entre 15 y $20 \mathrm{~cm}$ de diámetro y 0.35 metros de diámetro en tocones menores de $15 \mathrm{~cm}$.

Obteniéndose un resultado de parámetro de la mediana de 0.0001 lo cual indica que es altamente significativo. Con este resultado estadístico demuestra que influye bastante el diámetro de $15 \mathrm{~cm}$ del tocón en el crecimiento del diámetro de los rebrotes (tabla 3 ).

La prueba de t-Studen se efectuó en la comparación de mediana del incremento del diámetro de los rebrotes, dando un resultado de $2.125 \mathrm{~cm}$ de diámetro de los rebrotes en tocones entre 15 y $20 \mathrm{~cm}$. En tocones con diámetros menores $15 \mathrm{~cm}$, dio un resultado de $2.20 \mathrm{~cm}$, se obtuvo un parámetro de la mediana de 0.2530 , lo que indica que no hubo diferencia significativa en el incremento en diámetro para los rebrotes (tabla 3).
Análisis estadístico de altura del rebrote y diámetro del rebrote con respecto a la altura del tocón

Altura del rebrote. Según el resultado estadístico de t-Student, el incremento de la mediana en altura de los rebrotes varía de 1.88 metros en tocones con altura mayores de $20 \mathrm{~cm}$ a 2.30 metros en tocones con altura menores de $20 \mathrm{~cm}$, dando un resultado de parámetro de la mediana de 0.0396, es significativo, por que entre más se aleja el valor del parámetro será significativo, Esto indica que entre menor es el diámetro del tocón mayor será la altura de los rebrotes estadísticamente estudiados (tabla 2).

A través de una prueba de t-Student se compararon la mediana del diámetro del tocón con respecto al diámetro del rebrote y varía de $1.680 \mathrm{~cm}$ en tocones mayores de $20 \mathrm{~cm}$ y $0.6950 \mathrm{~cm}$ tocones menores de $20 \mathrm{~cm}$, dando un resultado del parámetro de la mediana de 0.0001 , esto indica que es altamente significativo en el incremento en diámetro, indicando que entre menor sea el tocón mayor será el incremento en diámetro de los rebrotes (tabla 2)
Tabla 3. Prueba de t-Sudent con respecto al incremento en altura de los rebrotes con variable del diámetro del tocón menor de $15 \mathrm{~cm}$ y entre $15-20 \mathrm{~cm}$, e incremento en diámetro de los ejes con la variable del diámetro del tocón menor de $15 \mathrm{~cm}$ y entre $15-20 \mathrm{~cm}$ finca El Plantel, 2011

\begin{tabular}{lcccc}
\hline Fuente de variación & $<15 \mathrm{~cm}$ & $15-20 \mathrm{~cm}$ & $<15 \mathrm{~cm}$ & $15-20 \mathrm{~cm}$ \\
\hline Parámetro & $\begin{array}{c}\text { Incremento altura } \\
\text { rebrote }\end{array}$ & $\begin{array}{c}\text { Incremento altura } \\
\text { rebrote }\end{array}$ & $\begin{array}{c}\text { Incremento } \\
\text { Diámetro re- } \\
\text { brote }\end{array}$ & $\begin{array}{c}\text { Incremento } \\
\text { Diámetro re- } \\
\text { brote }\end{array}$ \\
Desviación estándar & 1.026 & 1.385 & 2.522 & 1.654 \\
Mediana & 0.3500 & 1.800 & 2.200 & 2.125 \\
\hline
\end{tabular}

Sobrevivencia. Se contabilizaron 600 rebrotes al inicio del establecimiento de las parcelas, encontrándose en al cabo de siete meses una cantidad de 528 rebrotes vivos, la pérdida de estos rebotes es ocasionado por los daños mecánicos de los semovientes, resultando un valor del $88 \%$ de sobrevivencia, lo cual indica que es buena según la tabla de sobrevivencia de Centeno (1993). Realizando una comparación de la

Tabla 2. Prueba de t-Studen con respecto al incremento de altura de los ejes con la variable altura del tocón menor y mayor de $20 \mathrm{~cm}$, y diámetro de los rebrotes con la variable altura del tocón menor y mayor de $20 \mathrm{~cm}$ finca El Plantel, 2011

\begin{tabular}{ccccc}
\hline Fuente de variación & $>20 \mathrm{~cm}$ & $<20 \mathrm{~cm}$ & $>20 \mathrm{~cm}$ & $<20 \mathrm{~cm}$ \\
\hline Parámetro & $\begin{array}{c}\text { Incremento altura } \\
\text { rebrotes }\end{array}$ & $\begin{array}{c}\text { Incremento altura } \\
\text { rebrotes }\end{array}$ & $\begin{array}{c}\text { Incremento diáme- } \\
\text { tro rebrote }\end{array}$ & $\begin{array}{c}\text { Incremento diáme- } \\
\text { tro rebrotes }\end{array}$ \\
Desviación estándar & 1.793 & 1.826 & 1.123 & 1.648 \\
Mediana & 2.300 & 1.885 & 0.6950 & 1.680 \\
\hline
\end{tabular}

$\mathrm{P}<0.05 \quad 0.0396<0.05 \rightarrow$ significativo

$\mathrm{P}<0.05 \quad 0.0001<0.05 \rightarrow$ Altamente significativo 
sobrevivencia de las comunidades Las Marías, Las Carpas y Los Mangles del departamento de León obtuvieron un promedio del $83 \%$ de sobrevivencia, bastante similares los resultados en ambos estudios.

\section{Recomendaciones técnicas para el manejo de rebrotes} en plantaciones de Eucalyptus camaldulensis en la finca

El Plantel. Mantener la vigilancia con personal de campo y protección física de los rebrotes en los primeros años de desarrollo, para evitar daños mecánicos causados por semovientes.

Implementar el manejo silvicultural realizando raleo de los rebrotes no deseados que emergen consecutivamente después de la aplicación del tratamiento raleo, para evitar que los rebrotes seleccionados bajen el ritmo de desarrollo.

Aplicar el tratamiento poda en altura menor de $20 \mathrm{~cm}$ en tocones porque se demostró que es el más indicado para el desarrollo de rebrotes tanto en diámetro como en altura.

Realizar limpieza de material combustible, hierbas indeseables, y lianas en la plantación de rebrotes cada seis meses para evitar posibles conatos de incendios dentro de la plantación.
Realizar un plan de manejo que incluya el monitoreo de rebrotes en la plantación de eucalipto para dar seguimiento metódico aplicando los tratamientos silviculturales adecuados.

\section{CONCLUSIONES}

Los tratamientos silviculturales que se aplicaron en las parcelas establecidas en la finca El Plantel ejercen influencia en el incremento del crecimiento en diámetro y altura en los rebrotes, la limpieza de la maleza, eliminación de lianas y raleo de rebrotes, favoreciéndolos al no tener competencia los rebrotes seleccionados.

De acuerdo al análisis estadístico t-Studen tocón menor de $20 \mathrm{~cm}$ de altura, es mejor criterio para la realización de manejo de rebrotes en futuro.

La sobrevivencia en las parcelas establecidas independientemente de los cuatro tratamientos que se aplicaron, es buena de acuerdo a porcentajes establecidos en otros estudios que es del $80 \%$.

Se recomienda seguir aplicando tratamientos silviculturales de poda ya implementados en la plantación de eucalipto, para obtener un mejor rendimiento en el crecimiento tanto en diámetro y altura en el manejo de rebrotes.

\section{REFERENCIAS BIBLIOGRÁFICAS}

ADEFOR (Asociación de Desarrollo Forestal y Agropecuario de Occidente). 1995. Comportamiento de 25 procedencias de 3 especies forestales del género Eucalyptus (E. camaldulensis Dehn, E. maculata Hook. F. y E. tereticornis Sm.) En Chancay (Cajamarca, Perú). Informe de investigación $\mathrm{N}^{\circ} 5.24$ p.

Centeno. 1993. Inventario Nacional de Plantaciones Forestales de Nicaragua. 79 p.

Fernández R. s.f. Las bendiciones de un árbol maldito. (en línea). Consultado 22 feb 2011. Disponible en http:www.lacomunidad. elpais.com

González, B; Silva, C; Salgado, O. 2008. Evaluación de la capacidad de rebrotes de dos especies arbóreas en el bosque tropical seco, Nandarola Pacifico Sur. Universidad Nacional Agraria. Managua, NI. 8(11):57-61.

MARENA (Ministerio de Recursos Naturales y del Ambiente) / INAFOR (Instituto Nacional Forestal) 2002. Guía de Especies Forestales de Nicaragua. Managua, NI, Editora de Arte, S.A.

Pérez Mairena, M. 2004. Manejo de rebrotes en plantaciones de Eucalyptus camaldulensis, en las comunidades Las Marías, Los Mangles y Las Carpas, municipio de Telica, departamento de León, Nicaragua. Tesis Ing. Forestal. Universidad Nacional Agraria, Facultad de Recursos Naturales y del Ambiente. 49 p.

Somarriba Chang, M. 1989. Planificación conservacionista de la finca El Plantel, Masaya. (Tesis) Instituto Superior de Ciencias Agrarias ISCA. Managua, Nicaragua. 38 p.

Valdivia. 2006. Evaluación técnica de un sistema tradicional de cosecha en plantaciones de Eucalyptus globulus de corta rotación. (en línea). Consultado ene 2011. Disponible en: http:www.redalyc.udemex.mx 